\title{
BAN Working Frequency: a Trade-Off Between Antenna Efficiency and Propagation Losses
}

\author{
Luca Petrillo*, Theodoros Mavridis*, Julien Sarrazin ${ }^{\S}$, \\ Jean-Michel Dricot* Aziz Benlarbi-Delaî ${ }^{*}$, and Philippe De Doncker* \\ *Wireless Communication Group, Opera Department, Ecole Polytechnique de Bruxelles \\ Bruxelles, 1050 Belgium. Email: lpetrill@ulb.ac.be \\ §UPMC Univ Paris 06, UR2, L2E, F-75005, Paris, France. Email: julien.sarrazin@upmc.fr
}

\begin{abstract}
This communication addresses power issues at physical layer in Body Area Networks. Friis equation is discussed by taking into account body path loss and antenna efficiency from frequency ranging from $400 \mathrm{MHz}$ to $60 \mathrm{GHz}$. Then power balance is presented for fixed antenna dimensions and varying frequency in order to establish a balance.
\end{abstract}

Index Terms-Millimeter wave propagation; Surface waves; Wireless body sensor networks;

\section{INTRODUCTION}

Medical sector is facing great challenges nowadays. The capacity of healthcare system has not kept pace with the demand for emergency and trauma care. Emergency Departments today operate at or over capacity. Aging population requires also more health-care. In a period of economical crisis, efficiency of the medical system needs to be increased significantly.

Recent years have witnessed the emergence of wireless Body Area Networks (BANs) for medical applications. BANs are sensor networks that are embedded on the human body and provide useful health-care monitoring such as EEG (Electroencephalography), ECG (Electrocardiography), EMG (Electromyography), blood pressure, glucose... The use of wireless technology to interconnect sensors enables practical and seamless means to monitor patients. It thus can lead to more efficient patient's management in hospital and during mass-casualty disasters by automatically monitoring the vitals signs of many patients simultaneously to shorten patient's wait- times. If BANs are connected to the Internet through a cellular network for example, they can also achieve continuous monitoring. This aspect has great outcomes since it is the key for fighting disease with a preventing approach and detecting at an early stage when a person's health changes to the worse, thereby preventing emergency cases such as heart attacks. Continuous monitoring also represents a comfortable and effective economic way of taking care of age-related illnesses. Furthermore, these capabilities enable large-scale infield medical and behavioral studies.

Consequently, Medical BANs (MBANs) have a huge potential and are expected to become a successful medical system that will help in solving the medical crisis and also in creating a new market in medical ICT (Information and Communication Technologies).
A key success factor of such wireless sensor networks will be their potential to be autonomous in terms of energy. In fact, depending on the applications, sensors have to operate during a few hours up to a few years while relying on batteries or, as considering by on-going research studies, on micro energy harvesters or wireless power transfer. In any case, the amount of available energy is limited. This limitation becomes more crucial as sensors decrease in size, thereby limiting the room for battery. However, for patients to accept wearing such BANs, it is obvious that sensors should be as small as possible.

Consequently, this communication addresses the power issues at the physical layer by considering the propagation channel on the human body surface and the antenna characteristics. In particular, the trade-off between sensor miniaturization and operating frequency will be discussed. Some theoretical bounds will be drawn for the path gain between sensors of a given size and operating at a given frequency.

\section{BODY CHANNEL}

Body channel for BAN has been widely studied at frequency ranging from $400 \mathrm{MHz}$ to $60 \mathrm{GHz}$ and beyond. Path loss and shadowing have been investigated in different scenarios, for static and dynamic cases, for vertical or horizontal polarization. For our study, we are interested in giving a generalized Friis equation in order to compare the influence of the gain of the antennas and the spatial attenuation on the received power. Analytical expressions can be used for this scope. Friis equation is :

$$
P_{r}=P_{t}+G_{t}+G_{r}+20 \cdot \log _{10} \frac{\lambda}{4 \pi r}
$$

where $P_{t}$ and $P_{r}$ are radiated and received powers in $\mathrm{dBm}$, $G_{t}$ and $G_{r}$ are the gains of the antennas at the transmitting and receiving side in $\mathrm{dB}, \lambda$ is the wavelength in free space at the operating frequency and $r$ is the distance between transmitter and receiver. The term $20 \cdot \log _{10} \frac{\lambda}{4 \pi r}$ is the free-space path loss and it can be modified by taking into account the presence of the human body. For instance, in the case of planar (same side of the torso) on-body propagation, Norton equations can be used [?] [?]. In Fig. ?? we have traced body channel measurement reported in the literature [?], [?] and we have compared them with the modified Friis path-loss term : 


$$
20 \cdot \log _{10} \frac{\lambda}{4 \pi L(r)}
$$

where $L(r)$ is extracted from Norton formulations for an elementary dipole radiating above a conducting surface [?]. In that can be seen that (??) fits well measurement at $400 \mathrm{MHz}$, $2.45 \mathrm{GHz}$ and $60 \mathrm{GHz}$, even at distances $d<\lambda$. It is important to note that using Friis equation allows to consider antenna gain as well.

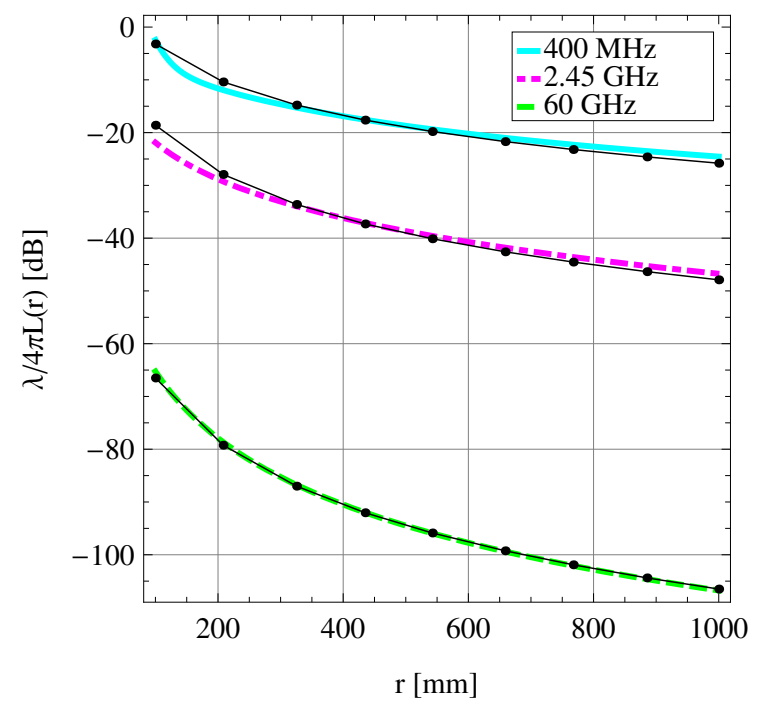

Fig. 1. Modified Friis path-loss term (solid lines) and path-loss model from measurements (dotted lines) for three frequencies in BAN scenario.

For on-body propagation around the torso, experimental results are well predicted by creeping wave formulations [?], [?]. Again, these formulations can be used to extend Friis formula for propagation around the body.

\section{Antenna Gain}

In BANs, antennas dimensions play a crucial role, since their miniaturization is a key factor for wearability. However, miniaturization can affect gain through efficiency, which, at lower frequencies, can result in a very low gain. Thus, the physical limitations of small antennas is determined using the well known Chu expression [?] in order to obtain the Q-factor of the small antenna as well as its input impedance. Using these characteristics, the appropriate matching network can be designed using a two-element L-section network. Knowing the antenna's Q-factor and its matching circuit, the overall efficiency $\eta_{s}$ can be then calculated using expressions given in [?]. Fig. 2 shows the efficiency $\eta_{s}$ of a small antenna of diameter $5 \mathrm{~mm}$ with respect to frequency. Results are given for several Q-factor values of the matching circuit components.

\section{CONCLUSION}

In this communication the propagation channel and the antenna gain are discussed for BANs ranging from $400 \mathrm{MHz}$ to $60 \mathrm{GHz}$. A modified Friis equation is proposed for taking

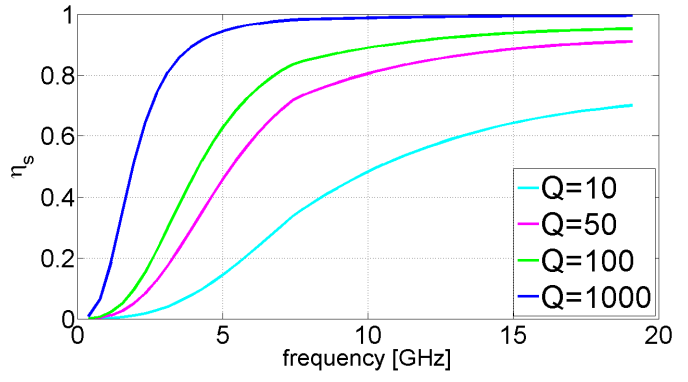

Fig. 2. Small antenna (diameter equal to $5 \mathrm{~mm}$ ) efficiency with respect to the frequency for different $\mathrm{Q}$ values of the matching circuit components.

into account the body channel and then a on-body path loss term is introduced and compared with measurement results reported in the literature. Antenna efficiency with respect to antenna dimensions and matching circuit $Q$ values is studied. In the conference speech, we will point out conclusions about the trade off between path-loss and efficiency at different frequencies of interest for BANs.

\section{REFERENCES}

[1] A. Lea et al., "Propagation between on-body antennas", Trans. Antennas and Prop., IEEE, Vol. 57, pp. 3619-3627, Nov. 2009.

[2] N. Chahat et al., "On-body Propagation at $60 \mathrm{GHz}$ ", Antennas and Propagation, IEEE Transactions on, vol. 61, pp. 1876-1888, 2013.

[3] C. Roblin et al., "Propagation channel models for BANs: an overview." COST 2100, Braunschweig, Germany, 16-18/02/2009.

[4] R. W. P. King et al., Cylindrical Antennas and Arrays. Cambridge, MA: Cambridge University Press, 2002.

[5] T. Alves et al., "Analytical Propagation Modeling of BAN Channels Based on the Creeping-Wave Theory", IEEE Trans. Antennas Propag., vol. 59, pp. 1269-1274, 2011.

[6] L. Petrillo, et al. "Analytical Creeping Waves Model At $60 \mathrm{GHz}$ for OnBody Communications." Proc. 2013 EUCAP, Goteborg, SW, 2013.

[7] L. J. Chu, "Physical Limitations of Omni-Directional Antennas", Journal of Applied Physics, Vol. 19, pp. 1163-1175, 1948.

[8] S. G. Smith, "Efficiency of Electrically Small Antennas Combined with Matching Networks", Trans. Antennas Propag., vol. 25, pp. 369-373, 1977. 